\title{
Aprendizagem Baseada em Equipe
}

Team Based Learning

Estrategia de Aprendizaje en Equipo

Denise Maria Almeida Bandeira ${ }^{1}$

Maria Alexsandra Silva ${ }^{2}$

Rosana Quintella B. Vilela ${ }^{3}$

\section{Resumo}

O mundo do trabalho contemporâneo exige a formação de profissionais com perfil crítico-reflexivo e capazes de trabalhar em equipes. Isto requer a formação de um profissional ativo e apto a aprender a aprender. Objetivo: descrever uma experiência com metodologias ativas, utilizando a estratégia da Aprendizagem Baseada em Equipe, do inglês Team Based Learning (TBL), em uma escola médica classificada, pela tipologia da Comissão de Avaliação das Escolas da área da Saúde (CAES), como inovadora com tendência tradicional. Método: $\mathrm{O}$ relato foi fundamentado na vivência e na análise das avaliações dos módulos mensais realizadas pelos estudantes e professores do setor de estudo de hematologia, da disciplina Saúde do Adulto e Idoso. Resultado: O estudo apontou a riqueza do TBL como oportunidade de praticar o uso de conceitos aplicado ao pensamento crítico, resolução de problemas, proporcionando maior interação entre alunos e professores, valorizando o trabalho em equipe, a busca ativa na construção do conhecimento e a capacidade de pactuação. Conclusão: O TBL é uma

${ }^{1}$ Mestrado em Saúde da Criança pela Universidade Federal de Alagoas. Docente adjunta da Faculdade de Medicina (FAMED) na Universidade Federal de Alagoas. Autor correspondente: End Campus A.C. Simões. Av. Lourival Melo Mota, s/n. Tabuleiro dos Martins.CEP:57072-900.Maceió - AL. E-mail: denisebandeira55@gmail.com

${ }^{2}$ Mestra em Clínica Médica (UNIFESP). Professora Assistente I da Universidade Federal de Alagoas (UFAL).

${ }^{3}$ Pós-doutorado pelo programa de pós-graduação em Ensino em Ciências da Saúde da UNIFESP. Docente titular da Universidade Federal de Alagoas.

Recebido: Nov/2016 - Aceito: Jan/2017. 
estratégia de ensino-aprendizagem bastante apropriada aos estudantes de medicina, na perspectiva de se permitir ensinar e aprender de forma colaborativa, por meio de atividades desafiadoras e refletir sobre as metodologias de ensino praticadas. Possibilita o envolvimento do docente em processos criativos comprometendoo com a construção de aprendizagens significativas, ativas e conscientes.

Descritores: Metodologia; Avaliação; Educação de Graduação em Medicina; Educação Médica.

\section{Abstract}

The contemporary world of work demands the qualification of professionals with a critical-reflexive profile, and with teamwork ability. This requires the education of active professionals, who are able to learn how to learn. Objective: Of this report is to describe an experience with active methodologies, using the strategy Team Based Learning (TBL), in a medical school classified by CAES (Committee of Assessment of Healthcare schools) as innovative with traditional tendency. Method: The report was based on experience, and on the analyses of the evaluation of monthly course modules by students and professors in the hematology study center, of the subject "Health for Adults and Elderly People”. Results: The study indicated the richness of TBL as an opportunity to put into practice the concepts applied to critical thinking and problem resolution, which provided greater interaction among students and professors. We valued team work, the active search for constructing knowledge, and the capacity to compromise. Conclusion: TBL is a teaching-learning strategy that is well suited to medical students, with a view to enabling them to teach and learn in a collaborative way, through challenging activities and to reflect on the teaching methodologies practiced. It allows the involvement of the teacher in creative processes, compromising him with the construction of meaningful, active and conscious learning.

Descriptors: Methodology; Evaluation; Undergraduate Medical Education; Medical Education.

\section{Resumen}

El mundo del trabajo contemporáneo exige la formación de profesionales 
críticos reflexivos y capaces de trabajar en equipo. Esto requiere la formación de un profesional activo y capaz de aprender a aprender. Objetivo: Este articulo pretende describir una vivencia con metodologías activas, utilizando la estrategia de aprendizaje en equipo, denominado en inglés como TBL (Team Based Learning), en una escuela de medicina clasificada como innovadora con tendencia tradicional, por el Comité de Evaluación de las Escuelas de la área de la Salud (CAES). Método: El informe se basó en la experiencia y en las análisis de los módulos mensuales de las evaluaciones, realizadas por estudiantes y profesores en la disciplina de la Salud de los Adultos y Ancianos, en el módulo de hematología. Resultados: El estudio señala la riqueza de la TBL como oportunidad para practicar el uso de conceptos aplicados al pensamiento crítico, solución de problemas, proporcionando una mayor interacción entre estudiantes y profesores, valorización del trabajo en equipo, la busca activa en la construcción de los conocimientos y la capacidad de acuerdo. Conclusión: TBL es una estrategia de aprendizaje de aprendizaje que está bien adaptada a los médicos de los médicos, con una vista para permitirles que se enseñan y aprenden en la forma de colaboración, a través de las actividades de reflexión $y$ reflexión sobre las metodologías practicadas. Se permite la participación del docente en procesos creativos, comprometiéndome con la construcción del significado, el aprendizaje activo y consciente.

Descriptores:

Metodologia;

Evaluación; Educación de Pregrado en Medicina; Educación Médica.

\section{Introdução}

Os novos paradigmas educacionais foram criados a partir das mudanças na prática docente em todos os níveis de ensino, mudanças essas que foram tornando-se necessárias pela força das modificações na sociedade.

A escola é referência para que o estudante elabore uma leitura de mundo baseada no conhecimento científico. Logo, o principal determinante no processo de elaboração dessa leitura, que é construção de conhecimento, é a ação pedagógica inserida nos currículos.

As metodologias de ensino como as ações do professor no sentido de organizar as atividades de ensino, a fim de que os alunos possam atingir os objetivos em relação a um conteúdo específico, tendo como resultado a 
assimilação dos conhecimentos e o desenvolvimento das capacidades cognitivas e operativas dos alunos ${ }^{(1)}$.

Ao longo dos anos, o estudo de metodologias ativas de EnsinoAprendizagem (MAEA) vem se intensificando com o surgimento de novas estratégias que podem favorecer a autonomia do educando, desde as mais simples àquelas que necessitam de uma readequação física e/ou tecnológica das instituições de ensino $^{(2,3)}$.

Como MAEA entende-se as atividades de ensino que ajudam as pessoas a assumir o controle de sua própria aprendizagem ${ }^{(4)}$. Bastos $(2006)^{(5)}$ as define como processos interativos de conhecimento, análise, estudos, pesquisas e decisões individuais ou coletivas, com a finalidade de encontrar soluções para um problema.

Neste sentido, Michael $(2006)^{(6)}$, em seu artigo de revisão, relata que não há um estudo definitivo para provar a eficiência das MAEA, dada a natureza dos fenômenos no trabalho. Mas, uma multiplicidade de fontes mostram que há evidências de que a aprendizagem com a abordagem ativa, centrada no aluno, tem melhores resultados na aprendizagem.

A Aprendizagem Baseada em
Equipes (ABE) ou Team-based learning (TBL) é um método de aprendizagem dinâmico, que proporciona um ambiente motivador e cooperativo, onde a produção coletiva é valorizada. Os estudantes se sentem motivados a participar, o que torna o ambiente de educação mais interessante, minimizando o desinteresse pelo aprendizado ${ }^{(3)}$.

Ciente de que as experiências realizadas em universidades podem servir para reflexões em diferentes instituições de ensino, e com a intencionalidade de compartilhar a reflexão da prática, para compreender suas possibilidades de transformação, este estudo teve como objetivo relatar as contribuições do TBL, como estratégia de ensino-aprendizagem, através de vivência em uma Universidade Pública do nordeste brasileiro.

\section{Método}

O TBL, introduzido nos anos 70 nas escolas de negócio( ${ }^{(7)}$, tem sua fundamentação teórica baseada no construtivismo, na qual o professor se torna um facilitador para a aprendizagem em um ambiente despido de autoritarismo e que privilegia a igualdade. As experiências e os conhecimentos prévios dos alunos são 
evocados na busca da aprendizagem significativa $^{(2,3)}$.

Para que a utilização desta metodologia aconteça com êxito, é preciso que o professor defina $\mathrm{o}$ conteúdo e o objetivo a ser alcançado pelos alunos neste conteúdo. Bem como, dividida a turma em grupos com 5 a 7 membros ${ }^{(8)}$.

$\mathrm{Na}$ primeira etapa (Preparação) o professor envia aos alunos materiais sobre o conteúdo que vai ser trabalhado, para que eles se preparem. Este material pode ser textos, exercícios, vídeos ou outra forma de apresentação do conteúdo ${ }^{(8)}$.

Em sala de aula, considerando o estudo antecipado, inicia-se a segunda etapa da estratégia aplicando um teste individual (Teste de Garantia de Estudo Prévio). Na sequência este mesmo teste é aplicado em grupo, momento em que os alunos discutem sobre o conteúdo, chegando a um consenso de suas $\operatorname{respostas}^{(8)}$.

Após o teste em grupo, eles recebem o feedback, e podem contestar as respostas das questões por escrito, com argumentação $\operatorname{adequada}^{(9)}$. O processo de garantia do estudo prévio é seguido pela devolutiva do professor, que pode, nesse momento, focar especificamente sobre o conteúdo que os alunos apresentaram dúvidas ${ }^{(8)}$.
$\mathrm{Na}$ terceira etapa, o professor aplica atividades/problemas relacionados ao conteúdo. Os estudantes devem ser desafiados a fazerem interpretação, inferências, análises ou síntese. Para avaliar a qualidade das respostas, podem ser utilizadas questões no formato de testes de múltipla escolha, verdadeiro ou falso ou questões abertas. A preparação para argumentar sobre a escolha realizada é muito importante para todas as equipes $^{(8)}$.

Esta etapa deve ser a mais longa e poderá ser repetida até que os objetivos de aprendizagem sejam contemplados, conforme o planejamento do professor e o tempo disponível para o curso ${ }^{8}$. O módulo ou unidade educacional em TBL pode ser concluído com esta fase, ou ainda, ser seguido por uma avaliação por pares, caracterizando a quarta etapa $^{(9)}$.

Esta estratégia foi aplicada no setor de estudo de Hematologia da disciplina de Saúde do Adulto e Idoso, do curso de medicina, durante dois semestres de 2015. O setor de estudo é contemplado com uma carga horária de três horas semanais por turma, por semestre. Todo o plano de ensino foi redesenhado e divido em seis módulos, e a cada módulo um novo processo de TBL era aplicado com a duração de 
seis horas (três horas/semana). Foram utilizadas duas turmas a cada semestre, com uma média de 22 alunos cada.

\section{Resultados}

Planejamento do TBL: O planejamento assume grande importância tendo em visto a necessidade de visualização de particularidades que serão imprescindíveis para o alcance do objetivo que se pretende.

Nesse momento foi contemplada a formação das equipes (docentes e discentes), a definição de objetivos de aprendizagem de cada módulo, o cronograma, a avaliação do estudante, a autoavaliação e a avaliação do módulo. A seguir será abordado cada item em particular, pela importância que possuem trazendo a tona o relato de experiência vivenciado.

Equipes: $\mathrm{O}$ grupo de trabalho foi composto por todos os professores (3) envolvidos no setor de estudo. Todos participaram de treinamento sobre a estratégia durante programa de capacitação docente da unidade acadêmica.

As equipes discentes foram constituídas de forma aleatória com 5 a 6 alunos cada. Ao longo do processo, por sugestão dos próprios estudantes, optou-se por modificar as equipes a cada módulo. Esta alteração mostrou-se positiva na visão dos docentes e discentes.

Definição de objetivos de aprendizagem: A determinação dos conceitos relevantes para a aprendizagem, em cada módulo, ocorreu em sintonia e auxílio da proposta curricular do curso.

Cronograma: As sessões ocorreram semanalmente, sendo necessárias duas semanas para cada módulo. No primeiro momento presencial foi realizado a Garantia de Preparo e na segunda semana foi desenvolvida a Aplicação de Conceitos e disponibilização de material para o próximo módulo (Preparação).

A avaliação do estudante: Toda pontuação do setor de estudo foi reservada para aplicação desta estratégia. $O$ peso de cada fase do processo foi pactuado com os estudantes, no primeiro dia de aula, a partir de uma proposta prévia dos professores. Essa proposta delimita o peso máximo e mínimo para cada um dos testes. A estratégia de desmembrar a nota em várias atividades estimulou uma maior presença às aulas.

O momento de apresentação da estratégia e pactuação dos pesos para cada teste, no primeiro dia de aula, 
mostrou-se fundamental para o compromisso do aluno com a estratégia e a aprendizagem. Observou-se um crescente no desempenho acadêmico dos alunos, ao longo do semestre.

A autoavaliação: Sobre a autoavaliação, Perrenoud (1999) ${ }^{(10)}$, afirma que se trata de formar o aluno para a regulação de seus próprios processos de pensamento e aprendizagem. Diante disso, a equipe docente optou também por esta avaliação na composição da estratégia. Para tanto, foi desenvolvida uma ficha com sugestão de indicadores para esta reflexão. Apesar do pequeno peso atribuído à autoavaliação (peso 1), os alunos mostraram-se entusiasmados com a inclusão da atividade na construção de sua avaliação final.

A avaliação do módulo: A necessidade de avaliar um projeto educativo parece ser óbvia, se pensarmos que ele surge de um problema e está carregado de intencionalidades; portanto é coerente observar, se as intenções foram alcançadas. Nesse sentido, o TBL foi avaliado, ao final de cada módulo, de forma muito positiva e entusiasmada pelos alunos e pelos docentes, agregando vantagens e novas habilidades a esta maneira de ensinar e aprender.

Figura 1 - Esquema do processo de aplicação do TBL

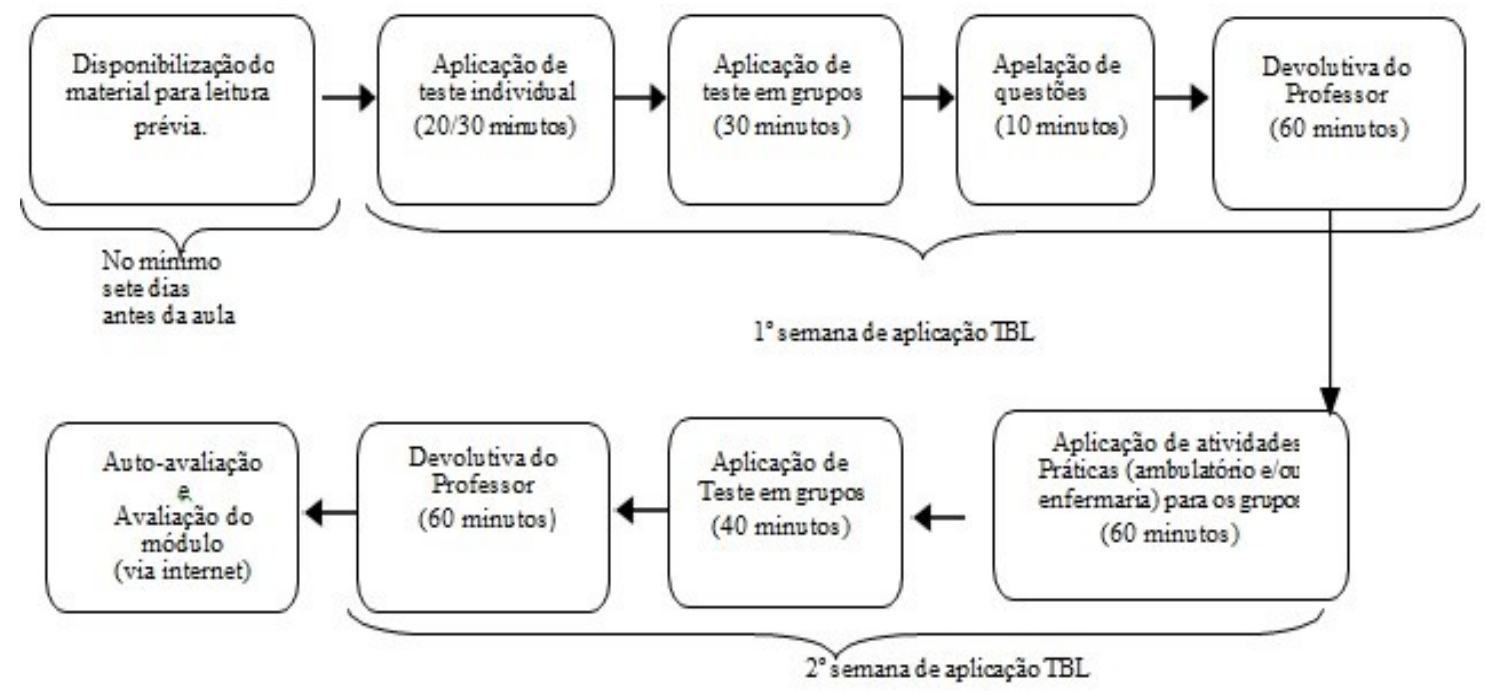

Os estudantes enfatizam que as aulas ficaram mais dinâmicas, participativas, produtivas e fazem com que eles estudem mais e interajam no grupo para resolver as atividades propostas. Sugeriram que outros 
professores explorassem essa estratégia nas aulas.

Os resultados desta avaliação colaboraram nos ajustes da técnica ao nosso contexto, e possibilitaram a divulgação da mesma na instituição.

Todo o processo utilizado para aplicação do TBL, nessa experiência, é descrito na figura acima (Figura 1).

Diante das constantes evoluções pedagógicas e tecnológicas, o TBL não trata-se de uma estratégia finalizada e imutável. As ferramentas pedagógicas devem sempre ser repensadas, recriadas e adequadas para contribuir com a formação dos futuros profissionais $^{(11)}$.

Nessa experiência o TBL mostrou-se uma ferramenta pedagógica que pode ser adaptada a vários contextos e conteúdos. $\mathrm{A}$ oportunidade de praticar o uso de conceitos aplicado ao pensamento crítico, resolução de problemas e a interação entre alunos e professores é garantida no processo, estimulando o trabalho em equipe e a capacidade de pactuação.

Observa-se que diante da possibilidade de participação ativa na discussão e negociação dos termos e efeitos da avaliação, o aluno que se autoavalia revela um grande envolvimento pessoal no processo de avaliação, gerindo e refletindo sobre o seu desempenho, tornando-se assim regulador da sua própria aprendizagem.

\section{Conclusão}

O TBL é uma estratégia de ensino-aprendizagem bastante apropriada aos estudantes de medicina, na perspectiva de se permitir ensinar e aprender de forma colaborativa, por meio de atividades desafiadoras e refletir sobre as metodologias de ensino praticadas. Possibilita o envolvimento do docente em processos criativos comprometendo-o com a construção de aprendizagens significativas, ativas e conscientes.

\section{Referências}

1. Libâneo JC. Didática, São Paulo: Cortez, 1994.

2. Bollela VR, Senger MH, Tourinho FV, Amaral E. Aprendizagem baseada em equipes: da teoria à prática. Medicina (Ribeirão Preto) 2014;47(3): 293-300.

3. Farias PAM, Martin ALAR, Cristo CS. Aprendizagem Ativa na Educação em Saúde: Percurso Histórico e Aplicações. RBEM. 2015;39(1): 14358.

4. Bransford JD, Brown AL, Cocking RR (Org.). Como as pessoas aprendem: cérebro, mente, experiência e escola, São Paulo (SP): SENAC, 2007. 
5. Bastos CC- Metodologias ativas. Educação e Medicina. 2006. Disponível em: http:/educacaoemedicina.blogspot .com.br/2006/02/metodologias-ativas. html>. [citado 04 abril 2016].

6. Michael J. Where's the evidence that active learning works? Adv Physiol Educ. 2006; 30: 159-67.

7. Burguess AW, McGregor DM, Mellis $\mathrm{CM}$, Applying established Guidelines to team-based learning programs in medical schools: A systematic review. Acad Med. 2014; 19:1-11.

8. Michaelsen LK. Getting Started with Team Based Learning. In: Michaelsen LK, Knight A B, Fink LD , editors. Team-Based Learning: A Transformative Use of Small Groups. Praeger; 2002.

9. Simonson SR. Making students do the thinking: team-based learning in a laboratory course. Adv Physiol Educ. 2014; 38(1): 49-55.

10. Perrenoud P. Avaliação: da excelência à regulação das aprendizagens - entre duas lógicas. Trad. Patrícia Chittoni Ramos. Porto Alegre: Artes Médicas Sul, 1999.

11. Dias RF. Team-based learning: fazendo os alunos pensarem "fora da caixa", os elementos essenciais para sua implantação. REBES. 2015; 5(1): 7581. 